\title{
Absolute tensor products, Kummer's formula and functional equations for multiple Hurwitz zeta functions
}

1. Introduction. Let

$$
Z_{j}(s) \cong \prod_{\varrho \in \mathbb{C}}((s-\varrho))^{m_{j}(\varrho)}:=\exp \left[-\left.\frac{\partial}{\partial w} \sum_{\varrho \in \mathbb{C}} \frac{m_{j}(\varrho)}{(s-\varrho)^{w}}\right|_{w=0}\right]
$$

be meromorphic functions expressed as regularized products, where $f(s) \cong$ $g(s)$ means that $f(s)=e^{Q(s)} g(s)$ for some $Q(s) \in \mathbb{C}[s]$. Their absolute tensor product is defined by

$$
Z_{1}(s) \otimes \cdots \otimes Z_{r}(s):=\prod_{\varrho_{1}, \ldots, \varrho_{r} \in \mathbb{C}}\left(\left(s-\varrho_{1}-\cdots-\varrho_{r}\right)\right)^{m\left(\varrho_{1}, \ldots, \varrho_{r}\right)},
$$

where

$$
m\left(\varrho_{1}, \ldots, \varrho_{r}\right):=m_{1}\left(\varrho_{1}\right) \cdots m_{r}\left(\varrho_{r}\right) \times \begin{cases}1 & \text { if } \operatorname{Im}\left(\varrho_{1}\right), \ldots, \operatorname{Im}\left(\varrho_{r}\right) \geq 0, \\ (-1)^{r-1} & \text { if } \operatorname{Im}\left(\varrho_{1}\right), \ldots, \operatorname{Im}\left(\varrho_{r}\right)<0, \\ 0 & \text { otherwise. }\end{cases}
$$

The absolute tensor product originates from Kurokawa $[\mathrm{K}]$. We refer to Manin $[\mathrm{M}]$ for an excellent survey.

Suppose that $Z_{1}(s), \ldots, Z_{r}(s)$ have properties of zeta functions, such as Euler product expressions and functional equations. Then their absolute tensor product $Z_{1}(s) \otimes \cdots \otimes Z_{r}(s)$ is also expected to have properties similar to those of usual zeta functions. We also expect that the (conjectural) generalized Euler product expression for the absolute tensor product of usual zeta functions $Z_{j}(s)$ would be related to absolute tensor products of Euler factors of $Z_{j}(s)$. These expectations are based on the analytic principle which is called the multiple explicit formula: see [A1, A2, KK3, KK4].

We consider the Hasse zeta functions $\zeta\left(s, \mathbb{F}_{p}\right):=\left(1-p^{-s}\right)^{-1}$ of finite fields $\mathbb{F}_{p}$. They can also be regarded as Euler factors of the Riemann

The author is supported by JSPS Research Fellowships for Young Scientists. 
zeta function. They have the following expression in terms of the (poly-) logarithm:

$$
\zeta\left(s, \mathbb{F}_{p}\right)=\exp \left[\sum_{n=1}^{\infty} \frac{p^{-n s}}{n}\right] \quad(\operatorname{Re}(s)>0) .
$$

The purpose of this paper is to give a generalization of (1.1) in the case of absolute tensor products of $\zeta\left(s, \mathbb{F}_{p}\right)$ :

Theorem 1. Let $p_{1}, \ldots, p_{k}$ be distinct prime numbers and $r_{1}, \ldots, r_{k}$ be positive integers. Then for $\operatorname{Re}(s)>0$ we have

$$
\begin{aligned}
\zeta\left(s, \mathbb{F}_{p_{1}}\right)^{\otimes r_{1}} \otimes \cdots \otimes \zeta\left(s, \mathbb{F}_{p_{k}}\right)^{\otimes r_{k}} \\
\cong \exp \left[\sum_{j=1}^{k} \sum_{n=1}^{\infty} \frac{1}{n}\left[g_{r_{j}}\left(\frac{1}{2 \pi i n} \frac{\partial}{\partial u}\right)\left(\frac{p_{j}^{-n s u}}{u \prod_{l \neq j}\left(e\left(n \frac{\log p_{j}}{\log p_{l}} u\right)-1\right)^{r_{l}}}\right)\right]_{u=1}\right],
\end{aligned}
$$

where $e(x):=e^{2 \pi i x}$ and

$$
g_{N}(z):= \begin{cases}\frac{(z-1)(z-2) \cdots(z-(N-1))}{(N-1) !} & \text { if } N \geq 2, \\ 1 & \text { if } N=1 .\end{cases}
$$

REMARK 1.1. The convergence of the summations depends on a consequence of Baker's result (see [B, Theorem 3.1]). That is, for distinct prime numbers $p, q$ there exists $c=c(p, q)>0$ such that

$$
\left\|n \frac{\log q}{\log p}\right\|^{-1}=O\left(n^{c}\right) \quad \text { as } n \rightarrow \infty,
$$

where $\|x\|:=\min _{m \in \mathbb{Z}}|x-m|$.

Remark 1.2. Theorem 1 was conjectured in [A2, Conjecture 5]. The following cases of Theorem 1 have been proved in earlier studies:

(1) $k=1([\mathrm{~K}])$,

(2) $r_{1}=\cdots=r_{k}=1([\mathrm{KK} 3, \mathrm{~A} 1, \mathrm{KW} 1])$,

(3) $k=2([\mathrm{~A} 2])$.

To prove Theorem 1, we give the corresponding expression for the (generalized) multiple sine function in Theorem 2 below. We recall the construction of the generalized multiple sine function introduced by Kurokawa-Ochiai [KO] and Kurokawa-Wakayama [KW2]. Let

$$
\zeta_{r}(s, z, \underline{\eta})=\sum_{n_{1}, \ldots, n_{r} \geq 0}\left(n_{1} \eta_{1}+\cdots+n_{r} \eta_{r}+z\right)^{-s}
$$

be the multiple Hurwitz zeta function introduced by Barnes [Bar], where $\eta:=\left(\eta_{1}, \ldots, \eta_{r}\right) \in\left(\mathbb{R}_{>0}\right)^{r}, \operatorname{Re}(z)>0$ and $\left|\arg \left(n_{1} \eta_{1}+\cdots+n_{r} \eta_{r}+z\right)\right|<\pi / 2$. The summation converges absolutely and uniformly on any compact subset of $\operatorname{Re}(s)>r$ and $\zeta_{r}(s, z, \underline{\eta})$ has an analytic continuation as a holomorphic 
function to $s \in \mathbb{C} \backslash\{1, \ldots, r\}$. Then, for $m \in \mathbb{Z}_{\geq 0}$, the generalized multiple gamma function $\Gamma_{r, m}(z, \underline{\eta})$ and the generalized multiple sine function $S_{r, m}(z, \eta)$ are defined by

$$
\begin{aligned}
\Gamma_{r, m}(z, \underline{\eta}) & :=\exp \left[\left.\frac{\partial}{\partial s} \zeta_{r}(s, z, \underline{\eta})\right|_{s=-m}\right], \\
S_{r, m}(z, \underline{\eta}) & :=\Gamma_{r, m}(z, \underline{\eta})^{-1} \Gamma_{r, m}\left(\eta_{1}+\cdots+\eta_{r}-z, \underline{\eta}\right)^{(-1)^{r+m}} .
\end{aligned}
$$

The multiple sine function $S_{r}(z, \underline{\eta})$ is given by $S_{r}(z, \eta):=S_{r, 0}(z, \underline{\eta})$. For its theory see [KK1]. While $S_{r}(z, \underline{\eta})$ is meromorphic in $z \in \mathbb{C}, S_{r, m}(z, \underline{\eta})$ cannot be extended meromorphically to all $z \in \mathbb{C}$ for any $m \geq 1$. We show Kummer's formula for the generalized multiple sine functions, which is a Fourier series type expansion for their logarithm:

Theorem 2. Suppose that $\omega_{1}, \ldots, \omega_{k}$ satisfy $\left\|n \omega_{l} / \omega_{j}\right\|^{-1}=O\left(e^{\varepsilon n}\right)$ as $n \rightarrow \infty$ for any $j, l(j \neq l)$ and any $\varepsilon>0$. Let $r_{1}, \ldots, r_{k} \in \mathbb{Z}_{>0}$ and $m \in \mathbb{Z}_{\geq 0}$. Put $r:=r_{1}+\cdots+r_{k}$ and $\underline{\omega}:=(\underbrace{\omega_{1}, \ldots, \omega_{1}}_{r_{1} \text { copies }}, \ldots, \underbrace{\omega_{k}, \ldots, \omega_{k}}_{r_{k} \text { copies }}) \in\left(\mathbb{R}_{>0}\right)^{r}$. Then:

(1) For $0<\operatorname{Re}(z)<r_{1} \omega_{1}+\cdots+r_{k} \omega_{k}, \operatorname{Im}(z)>0$ we have

$$
\begin{aligned}
S_{r, m}(z, \underline{\omega})= & \exp \left[m ! \sum_{j=1}^{k}(-1)^{r_{j}} \sum_{n=1}^{\infty} \frac{1}{n}\left(\frac{\omega_{j}}{2 \pi i n}\right)^{m}\right. \\
& \times\left[g_{r_{j}}\left(\frac{1}{2 \pi i n} \frac{\partial}{\partial u}\right)\left(\frac{e\left(\frac{n z}{\omega_{j}} u\right)}{u^{m+1} \prod_{l \neq j}\left(1-e\left(\frac{n \omega_{l}}{\omega_{j}} u\right)\right)^{r_{l}}}\right)\right]_{u=1} \\
& \left.+\pi i \zeta_{r}(-m, z, \underline{\omega})\right] .
\end{aligned}
$$

The right hand side is holomorphic in $\operatorname{Im}(z)>0$.

(2) For $0<\operatorname{Re}(z)<r_{1} \omega_{1}+\cdots+r_{k} \omega_{k}, \operatorname{Im}(z)<0$ we have

$$
\begin{aligned}
S_{r, m}(z, \underline{\omega})= & \exp \left[(-1)^{m} m ! \sum_{j=1}^{k}(-1)^{r_{j}} \sum_{n=1}^{\infty} \frac{1}{n}\left(\frac{\omega_{j}}{2 \pi i n}\right)^{m}\right. \\
& \times\left[g_{r_{j}}\left(-\frac{1}{2 \pi i n} \frac{\partial}{\partial u}\right)\left(\frac{e\left(-\frac{n z}{\omega_{j}} u\right)}{u^{m+1} \prod_{l \neq j}\left(1-e\left(-\frac{n \omega_{l}}{\omega_{j}} u\right)\right)^{r_{l}}}\right)\right]_{u=1} \\
& \left.-\pi i \zeta_{r}(-m, z, \underline{\omega})\right] .
\end{aligned}
$$

The right hand side is holomophic in $\operatorname{Im}(z)<0$.

Remark 1.3. Theorem 2(1) with $m=0$ was conjectured in [A2, Conjecture 4]. 
The key point to prove Theorem 2 is to show the functional equation for a suitable sum of multiple Hurwitz zeta functions as follows:

Theorem 3. Let $\omega_{1}, \ldots, \omega_{k}, r_{1}, \ldots, r_{k}, r$ and $\underline{\omega}$ be as in Theorem 2. Then:

(1) For $0<\operatorname{Re}(z)<r_{1} \omega_{1}+\cdots+r_{k} \omega_{k}, \operatorname{Im}(z)>0$ and $\operatorname{Re}(s)<1$ we have

$$
\begin{aligned}
& e^{\pi i s} \zeta_{r}(s, z, \underline{\omega})+(-1)^{r-1} \zeta_{r}\left(s, r_{1} \omega_{1}+\cdots+r_{k} \omega_{k}-z, \underline{\omega}\right) \\
&=-\frac{2 \pi i}{\Gamma(s)} \sum_{j=1}^{k} \frac{(-1)^{r_{j}}}{\omega_{j}} \sum_{n=1}^{\infty}\left(\frac{2 \pi i n}{\omega_{j}}\right)^{s-1} \\
& \times\left[g_{r_{j}}\left(\frac{1}{2 \pi i n} \frac{\partial}{\partial u}\right)\left(\frac{u^{s-1} e\left(\frac{n z}{\omega_{j}} u\right)}{\prod_{l \neq j}\left(1-e\left(\frac{n \omega_{l}}{\omega_{j}} u\right)\right)^{r_{l}}}\right)\right]_{u=1},
\end{aligned}
$$

where $\arg \left(2 \pi i n / \omega_{j}\right)=\pi / 2$. The summations over $n$ converge absolutely and uniformly on any compact subset of $\left\{(s, z) \in \mathbb{C}^{2}: \operatorname{Im}(z)\right.$ $>0\}$.

(2) For $0<\operatorname{Re}(z)<r_{1} \omega_{1}+\cdots+r_{k} \omega_{k}, \operatorname{Im}(z)<0$ and $\operatorname{Re}(s)<1$ we have

$$
\begin{aligned}
\zeta_{r}(s, z, \underline{\omega})+( & -1)^{r-1} e^{\pi i s} \zeta_{r}\left(s, r_{1} \omega_{1}+\cdots+r_{k} \omega_{k}-z, \underline{\omega}\right) \\
= & -\frac{2 \pi i}{\Gamma(s)} \sum_{j=1}^{k} \frac{(-1)^{r_{j}}}{\omega_{j}} \sum_{n=1}^{\infty}\left(\frac{2 \pi i n}{\omega_{j}}\right)^{s-1} \\
& \times\left[g_{r_{j}}\left(-\frac{1}{2 \pi i n} \frac{\partial}{\partial u}\right)\left(\frac{u^{s-1} e\left(-\frac{n z}{\omega_{j}} u\right)}{\prod_{l \neq j}\left(1-e\left(-\frac{n \omega_{l}}{\omega_{j}} u\right)\right)^{r_{l}}}\right)\right]_{u=1} .
\end{aligned}
$$

The summations over $n$ converge absolutely and uniformly on any compact subset of $\left\{(s, z) \in \mathbb{C}^{2}: \operatorname{Im}(z)<0\right\}$.

We will also obtain the functional equation for multiple Hurwitz zeta functions:

Theorem 4. Let $\omega_{1}, \ldots, \omega_{k}, r_{1}, \ldots, r_{k}, r$ and $\underline{\omega}$ be as in Theorem 2. In addition, suppose that for any $j, l(j \neq l)$ there exists $c_{j, l}>0$ such that $\left\|n \omega_{l} / \omega_{j}\right\|^{-1}=O\left(n^{c_{j, l}}\right)$ as $n \rightarrow \infty$. Then, for $0<z<r_{1} \omega_{1}+\cdots+r_{k} \omega_{k}$ and $\operatorname{Re}(s)<A$ we have

$$
\begin{aligned}
\zeta_{r}(s, z, \underline{\omega})= & -\Gamma(1-s) \sum_{j=1}^{k} \frac{(-1)^{r_{j}}}{\omega_{j}} \sum_{n \in \mathbb{Z} \backslash\{0\}}\left(\frac{2 \pi i n}{\omega_{j}}\right)^{s-1} \\
& \times\left[g_{r_{j}}\left(\frac{1}{2 \pi i n} \frac{\partial}{\partial u}\right)\left(\frac{u^{s-1} e\left(\frac{n z}{\omega_{j}} u\right)}{\prod_{l \neq j}\left(1-e\left(\frac{n \omega_{l}}{\omega_{j}} u\right)\right)^{r_{l}}}\right)\right]_{u=1},
\end{aligned}
$$


where $\arg \left(2 \pi i n / \omega_{j}\right)= \pm \pi / 2$ and $A \in \mathbb{R}$ is a constant given explicitly in terms of $r_{j}$ and $c_{j, l}$.

REMARK 1.4. To guarantee the convergence of the summations over $n$ on the right hand side of the equation in Theorem 4 , we need the stronger assumption " $\left\|n \omega_{l} / \omega_{j}\right\|^{-1}=O\left(n^{c_{j, l}}\right)$ as $n \rightarrow \infty$ " in comparison with Theorems 2 and 3.

Remark 1.5. From Theorem 4 we can obtain Kummer's formula for the generalized multiple gamma function $\Gamma_{r, m}(z, \underline{\omega})$ for sufficiently large $m$. But in the setting of Theorem 4 with $m=0$, which is the most interesting case, it seems difficult to obtain Kummer's formula for the multiple gamma function $\Gamma_{r}(z, \underline{\omega})=\Gamma_{r, 0}(z, \underline{\omega})$ because we encounter the problem about the validity of Theorem 4 at $s=0$. For some concrete $\underline{\omega}$ Koyama and Kurokawa [KK2] obtained Kummer's formula for multiple gamma functions.

\section{Functional equations for multiple Hurwitz zeta functions. In} this section we prove Theorems 3 and 4 .

First we sketch the proof. Let $\omega_{1}, \ldots, \omega_{k}, r_{1}, \ldots, r_{k}, r, \underline{\omega}$ be as in Theorem 2. We use the following contour integral expression for multiple Hurwitz zeta functions:

$$
\begin{aligned}
\zeta_{r}(s, z, \underline{\omega})=-\frac{\Gamma(1-s)}{2 \pi i} \int_{C_{\varepsilon}} \frac{e^{-z t}}{\left(1-e^{-\omega_{1} t}\right)^{r_{1}} \cdots\left(1-e^{-\omega_{k} t}\right)^{r_{k}}}(-t)^{s-1} d t \\
\quad\left(s \in \mathbb{C} \backslash \mathbb{Z}_{>0}, \operatorname{Re}(z)>0\right)
\end{aligned}
$$

where $0<\varepsilon<\min \left\{2 \pi / \omega_{1}, \ldots, 2 \pi / \omega_{k}\right\}$ is a fixed number; $C_{\varepsilon}$ is the union of the interval from $+\infty$ to $\varepsilon$, the set $\left\{\varepsilon e^{i \theta}: \theta\right.$ from 0 to $\left.2 \pi\right\}$ and the interval from $\varepsilon$ to $+\infty ;(-t)^{s-1}=e^{(s-1) \log (-t)}$ and $\log (-t)$ takes a real value at $t=-\varepsilon$ on $C_{\varepsilon}$. We put $C_{\varepsilon, R, T}:=\bigcup_{j=1}^{6} C^{(j)}$ with

$$
\begin{aligned}
C^{(1)}:= & \text { the interval from } R e^{2 \pi i} \text { to } \varepsilon e^{2 \pi i} \\
& \cup\left\{\varepsilon e^{i \theta}: \theta \text { from } 2 \pi \text { to } 0\right\} \cup[\varepsilon, R], \\
C^{(2)}:= & \{R+i u: u \text { from } 0 \text { to } T\}, \\
C^{(3)}:= & \{u+i T: u \text { from } R \text { to }-R\}, \\
C^{(4)}:= & \{-R+i u: u \text { from } T \text { to }-T\}, \\
C^{(5)}:= & \{u-i T: u \text { from }-R \text { to } R\}, \\
C^{(6)}:= & \{R+i u: u \text { from }-T \text { to } 0\},
\end{aligned}
$$

where $T \in \mathbb{R}_{>\varepsilon} \backslash \bigcup_{j=1}^{k}\left(2 \pi / \omega_{j}\right) \mathbb{Z}$ and $R \in \mathbb{R}_{>\varepsilon}$. By the residue theorem, we have 


$$
\begin{aligned}
& \frac{1}{2 \pi i} \int_{C_{\varepsilon, R, T}} \frac{e^{-z t}}{\left(1-e^{-\omega_{1} t}\right)^{r_{1}} \cdots\left(1-e^{-\omega_{k} t}\right)^{r_{k}}}(-t)^{s-1} d t \\
& =\sum_{j=1}^{k} \sum_{\substack{|n|<\omega_{j} T / 2 \pi \\
n \neq 0}} \operatorname{Res}_{t=2 \pi i n / \omega_{j}} \frac{e^{-z t}}{\left(1-e^{-\omega_{1} t}\right)^{r_{1}} \cdots\left(1-e^{-\omega_{k} t}\right)^{r_{k}}}(-t)^{s-1} .
\end{aligned}
$$

Calculating the residue and taking the limit as $R \rightarrow \infty$ and $T \rightarrow \infty$, we will prove Theorems 3 and 4 . Strictly speaking, in the proof of Theorem 3 before taking the limit as $T \rightarrow \infty$ we will find the cancellation between divergent terms.

We begin the detailed proof. First we calculate the residue in (2.1):

Lemma 2.1. Let $\omega_{1}, \ldots, \omega_{k}, r_{1}, \ldots, r_{k}$ be as in Theorem $2, n \in \mathbb{Z} \backslash\{0\}$ and $z, s \in \mathbb{C}$. Then

$$
\begin{aligned}
& \text { (2.2) } \operatorname{Res}_{t=2 \pi i n / \omega_{j}} \frac{e^{-z t}}{\left(1-e^{-\omega_{1} t}\right)^{r_{1}} \cdots\left(1-e^{-\omega_{k} t}\right)^{r_{k}}}(-t)^{s-1} \\
& =\frac{(-1)^{r_{j}+1}}{\omega_{j}}\left(-\frac{2 \pi i n}{\omega_{j}}\right)^{s-1}\left[g_{r_{j}}\left(-\frac{1}{2 \pi i n} \frac{\partial}{\partial u}\right)\left(\frac{u^{s-1} e\left(-\frac{n z}{\omega_{j}} u\right)}{\prod_{l \neq j}\left(1-e\left(-\frac{n \omega_{l}}{\omega_{j}} u\right)\right)^{r_{l}}}\right)\right]_{u=1},
\end{aligned}
$$

where $\arg \left(-2 \pi i n / \omega_{j}\right)= \pm \pi / 2$.

To prove the lemma, we recall the multiple Bernoulli polynomial $B_{r, m}(x)$ ([N, Section 5, Chapter 6]). It is defined by the generating function as

$$
e^{x t}\left(\frac{t}{e^{t}-1}\right)^{r}=\sum_{m=0}^{\infty} \frac{B_{r, m}(x)}{m !} t^{m} \quad(|t|<2 \pi),
$$

and has the following property (see [N, (86), p. 147]):

$$
\frac{B_{r, m}(x)}{m !}=g_{r}^{(r-1-m)}(x) \quad(m=0,1, \ldots, r-1),
$$

where $g_{r}^{(k)}$ denotes the $k$ th derivative of $g_{r}$.

Proof of Lemma 2.1. Changing $t \mapsto t+2 \pi i n / \omega_{j}$, the left hand side of (2.2) equals

$$
\begin{aligned}
& e\left(-\frac{n}{\omega_{j}} z\right) \\
\times & \operatorname{Res}_{t=0}\left\{\frac{e^{-z t}}{\left(1-e^{-\omega_{j} t}\right)^{r_{j}}} \frac{1}{\prod_{l \neq j}\left(1-e^{-\omega_{l}\left(t+2 \pi i n / \omega_{j}\right)}\right)^{r_{l}}}\left(-t-\frac{2 \pi i n}{\omega_{j}}\right)^{s-1}\right\} .
\end{aligned}
$$

We expand each term around $t=0$. Replacing $x$ with $z / \omega_{j}, t$ with $-\omega_{j} t$ and $r$ with $r_{j}$ in (2.3), we have

$$
\frac{e^{-z t}}{\left(1-e^{-\omega_{j} t}\right)^{r_{j}}}=\frac{1}{\left(\omega_{j} t\right)^{r_{j}}} \sum_{m=0}^{\infty}(-1)^{m} \frac{B_{r_{j}, m}\left(z / \omega_{j}\right)}{m !} \omega_{j}^{m} t^{m} .
$$


Next we deal with the second term in braces in (2.5). For $|t|<2 \pi / \omega_{j}$ Taylor's theorem gives

$$
\begin{aligned}
& \frac{1}{\prod_{l \neq j}\left(1-e^{\left.-\omega_{l}\left(t+2 \pi i n / \omega_{j}\right)\right)^{r_{l}}}\right.} \\
& \quad=\sum_{m=0}^{\infty} \frac{1}{m !}\left[\frac{d^{m}}{d u^{m}}\left(\frac{1}{\prod_{l \neq j}\left(1-e^{-\omega_{l}\left(u+2 \pi i n / \omega_{j}\right)}\right)^{r_{l}}}\right)\right]_{u=0} t^{m} \\
& =\sum_{m=0}^{\infty} \frac{1}{m !}\left(\frac{\omega_{j}}{2 \pi i n}\right)^{m}\left[\frac{d^{m}}{d u^{m}}\left(\frac{1}{\prod_{l \neq j}\left(1-e\left(-\frac{n \omega_{l}}{\omega_{j}} u\right)\right)^{r_{l}}}\right)\right]_{u=1} t^{m} .
\end{aligned}
$$

Here, in the second equation we changed $u \mapsto \frac{2 \pi i n}{\omega_{j}}(u-1)$.

Next we treat the third term in braces in (2.5). We remark that $\arg \left(-t-2 \pi i n / \omega_{j}\right)$ takes a value near $-\pi / 2$ or $\pi / 2$ if $|t|$ is sufficiently small. Hence

$$
\begin{aligned}
\left(-t-\frac{2 \pi i n}{\omega_{j}}\right)^{s-1} & =\left(-\frac{2 \pi i n}{\omega_{j}}\right)^{s-1}\left(1+\frac{\omega_{j} t}{2 \pi i n}\right)^{s-1} \\
& =\left(-\frac{2 \pi i n}{\omega_{j}}\right)^{s-1} \sum_{m=0}^{\infty} g_{m+1}(s)\left(\frac{\omega_{j}}{2 \pi i n}\right)^{m} t^{m}
\end{aligned}
$$

where $\arg \left(-2 \pi i n / \omega_{j}\right)= \pm \pi / 2$.

Applying (2.6)-(2.8) to (2.5), we obtain

$$
\begin{aligned}
\operatorname{Res}_{t=} & 2 \pi i n / \omega_{j} \\
= & e\left(-\frac{n}{\omega_{j}} z\right) \frac{1}{\left(1-e^{-\omega_{1} t}\right)^{r_{1}} \cdots\left(1-e^{-\omega_{k} t}\right)^{r_{k}}}\left(-\frac{2 \pi i n}{\omega_{j}}\right)^{s-1} \\
& \times \sum_{\substack{m_{1}, m_{2}, m_{3} \geq 0 \\
m_{1}+m_{2}+m_{3}=r_{j}-1}}(-1)^{m_{1}} \frac{B_{r_{j}, m_{1}}\left(z / \omega_{j}\right)}{m_{1} !} \frac{1}{m_{2} !} \frac{1}{(2 \pi i n)^{m_{2}+m_{3}}} \\
& \times\left[\frac{d^{m_{2}}}{d u^{m_{2}}}\left(\frac{1}{\prod_{l \neq j}\left(1-e\left(-\frac{n \omega_{l}}{\omega_{j}} u\right)\right)^{r_{l}}}\right)\right]_{u=1} g_{m_{3}+1}(s) .
\end{aligned}
$$

Next we deal with the right hand side of (2.2). We put

$$
g_{r_{j}}(z)=\sum_{m=0}^{r_{j}-1} a_{r_{j}, m} z^{m} \quad\left(a_{r_{j}, m}=g_{r_{j}}^{(m)}(0) / m !\right) .
$$

Then by the Leibniz rule we have 


$$
g_{r_{j}}\left(-\frac{1}{2 \pi i n} \frac{\partial}{\partial u}\right)\left(\frac{u^{s-1} e\left(-\frac{n z}{\omega_{j}} u\right)}{\prod_{l \neq j}\left(1-e\left(-\frac{n \omega_{l}}{\omega_{j}} u\right)\right)^{r_{l}}}\right)
$$

$$
=\sum_{m=0}^{r_{j}-1} a_{r_{j}, m}\left(-\frac{1}{2 \pi i n}\right)^{m}
$$$$
\times \sum_{\substack{m_{2}, m_{3} \geq 0 \\ m_{2}+m_{3} \leq m}} \frac{m !}{\left(m-m_{2}-m_{3}\right) ! m_{2} ! m_{3} !}\left\{\left(-\frac{2 \pi i n}{\omega_{j}} z\right)^{m-m_{2}-m_{3}} e\left(-\frac{n z}{\omega_{j}} u\right)\right\}
$$$$
\times \frac{d^{m_{2}}}{d u^{m_{2}}}\left(\frac{1}{\prod_{l \neq j}\left(1-e\left(-\frac{n \omega_{l}}{\omega_{j}} u\right)\right)^{r_{l}}}\right)\left\{(s-1) \cdots\left(s-m_{3}\right) u^{s-m_{3}-1}\right\}
$$

$$
\begin{aligned}
& =\sum_{\substack{m_{2}, m_{3} \geq 0 \\
m_{2}+m_{3} \leq r_{j}-1}} \sum_{m=m_{2}+m_{3}}^{r_{j}-1} \ldots \\
& =e\left(-\frac{n z}{\omega_{j}} u\right)
\end{aligned}
$$

$$
\begin{aligned}
& \times \sum_{\substack{m_{2}, m_{3} \geq 0 \\
m_{2}+m_{3} \leq r_{j}-1}}\left(\sum_{m=m_{2}+m_{3}}^{r_{j}-1} a_{r_{j}, m} \frac{m !}{\left(m-m_{2}-m_{3}\right) !}\left(\frac{z}{\omega_{j}}\right)^{m-m_{2}-m_{3}}\right) \frac{1}{m_{2} !} \\
& \times\left(-\frac{1}{2 \pi i n}\right)^{m_{2}+m_{3}} \frac{d^{m_{2}}}{d u^{m_{2}}}\left(\frac{1}{\prod_{l \neq j}\left(1-e\left(-\frac{n \omega_{l}}{\omega_{j}} u\right)\right)^{r_{l}}}\right) g_{m_{3}+1}(s) u^{s-m_{3}-1} .
\end{aligned}
$$

Since it follows from (2.10) and (2.4) that

$$
\begin{aligned}
\sum_{m=m_{2}+m_{3}}^{r_{j}-1} a_{r_{j}, m} \frac{m !}{\left(m-m_{2}-m_{3}\right) !} z^{m-m_{2}-m_{3}} & =g_{r_{j}}^{\left(m_{2}+m_{3}\right)}(z) \\
& =\frac{B_{r_{j}, r_{j}-1-m_{2}-m_{3}}(z)}{\left(r_{j}-1-m_{2}-m_{3}\right) !},
\end{aligned}
$$

(2.11) equals (putting $m_{1}=r_{j}-1-m_{2}-m_{3}$ )

$$
\begin{aligned}
& (-1)^{r_{j}-1} e\left(-\frac{n z}{\omega_{j}} u\right) \sum_{\substack{m_{1}, m_{2}, m_{3} \geq 0 \\
m_{1}+m_{2}+m_{3}=r_{j}-1}}(-1)^{m_{1}} \frac{B_{r_{j}, m_{1}}\left(z / \omega_{j}\right)}{m_{1} !} \frac{1}{m_{2} !} \\
& \times \frac{1}{(2 \pi i n)^{m_{2}+m_{3}}} \frac{d^{m_{2}}}{d u^{m_{2}}}\left(\frac{1}{\prod_{l \neq j}\left(1-e\left(-\frac{n \omega_{l}}{\omega_{j}} u\right)\right)^{r_{l}}}\right) g_{m_{3}+1}(s) u^{s-m_{3}-1} .
\end{aligned}
$$

Comparing (2.9) to (2.12) with $u=1$, we obtain the desired result. 
Next, we restrict $z$ to $0<\operatorname{Re}(z)<r_{1} \omega_{1}+\cdots+r_{k} \omega_{k}$ and take the limit as $R \rightarrow \infty$ in (2.1). Then easy estimates give $\left|\int_{C^{(j)}}\right| \rightarrow 0$ as $R \rightarrow \infty$ for $j=2,4,6$. Thus, we obtain

Lemma 2.2. Let $\omega_{1}, \ldots, \omega_{k}, r_{1}, \ldots, r_{k}$ be as in Theorem $2, s \in \mathbb{C}$ and $T \in \mathbb{R}_{>\varepsilon} \backslash \bigcup_{j=1}^{k}\left(2 \pi / \omega_{j}\right) \mathbb{Z}$. Then for $0<\operatorname{Re}(z)<r_{1} \omega_{1}+\cdots+r_{k} \omega_{k}$ we have

$$
\begin{aligned}
\frac{1}{2 \pi i}\left(-\int_{C_{\varepsilon}}+\int_{C^{(7)}}+\right. & \left.\int_{C^{(8)}}\right) \frac{e^{-z t}}{\left(1-e^{-\omega_{1} t}\right)^{r_{1}} \cdots\left(1-e^{-\omega_{k} t}\right)^{r_{k}}}(-t)^{s-1} d t \\
= & -\sum_{j=1}^{k} \frac{(-1)^{r_{j}}}{\omega_{j}} \sum_{\substack{|n|<\omega_{j} T / 2 \pi \\
n \neq 0}}\left(\frac{2 \pi i n}{\omega_{j}}\right)^{s-1} \\
& \times\left[g_{r_{j}}\left(\frac{1}{2 \pi i n} \frac{\partial}{\partial u}\right)\left(\frac{u^{s-1} e\left(\frac{n z}{\omega_{j}} u\right)}{\prod_{l \neq j}\left(1-e\left(\frac{n \omega_{l}}{\omega_{j}} u\right)\right)^{r_{l}}}\right)\right]_{u=1},
\end{aligned}
$$

where

$$
\begin{aligned}
& C^{(7)}:=\{u+i T: u \text { from } \infty \text { to }-\infty\}, \\
& C^{(8)}:=\{u-i T: u \text { from }-\infty \text { to } \infty\},
\end{aligned}
$$

$|\arg (-t)|<\pi$ and $\arg \left(2 \pi i n / \omega_{j}\right)= \pm \pi / 2$.

Next, we consider the limit as $T \rightarrow \infty$. But the integrand in Lemma 2.2 has poles at $t \in i \mathbb{R}$. Hence, we choose a good sequence $T_{1} \leq \cdots \leq T_{N} \leq$ $\cdots \rightarrow \infty$, put $T=T_{N}$ and take the limit $N \rightarrow \infty$. The following lemma guarantees the existence of good sequences:

Lemma 2.3. Let $\omega_{1}, \ldots, \omega_{k}, r_{1}, \ldots, r_{k}$ be as in Theorem 2. Then there exists $C>0$ such that for any $T>0$ we can choose $T_{0} \in(T, T+1)$ satisfying

$$
\left|\frac{1}{\left(1-e^{-\omega_{1} t}\right)^{r_{1}} \cdots\left(1-e^{-\omega_{k} t}\right)^{r_{k}}}\right| \leq C
$$

for all $t=u \pm i T_{0}$ with $-1 \leq u \leq 1$.

Proof. For $t=u+i v$ with $-1 \leq u \leq 1$ and $v \in \mathbb{R}$ we have

$$
\begin{aligned}
\left|1-e^{-\omega_{j} t}\right| & =\left\{\left(1-e^{-\omega_{j} u} \cos \left(\omega_{j} v\right)\right)^{2}+e^{-2 \omega_{j} u} \sin ^{2}\left(\omega_{j} v\right)\right\}^{1 / 2} \\
& \geq e^{-\omega_{j} u}\left|\sin \left(\omega_{j}|v|\right)\right| \geq e^{-\omega_{j}} \sin \left(\pi\left\|\omega_{j}|v| / \pi\right\|\right) .
\end{aligned}
$$

Hence we have

$$
\left|\frac{1}{\left(1-e^{-\omega_{1} t}\right)^{r_{1}} \cdots\left(1-e^{-\omega_{k} t}\right)^{r_{k}}}\right| \leq \frac{e^{r_{1} \omega_{1}+\cdots+r_{k} \omega_{k}}}{\prod_{j=1}^{k} \sin ^{r_{j}}\left(\pi\left\|\omega_{j}|v| / \pi\right\|\right)} .
$$

Since the number of $v \in(T, T+1)$ which satisfy $\omega_{j} v / \pi \in \mathbb{Z}$ for some $j$ is bounded above uniformly in $T$, there exists $\delta>0$, which does not depend on $T$, and there exists $T_{0} \in(T, T+1)$ such that $\delta \leq\left\|\omega_{j} T_{0} / \pi\right\| \leq 1 / 2$ for any $j$. Applying this to (2.13) with $v= \pm T_{0}$ completes the proof. 
Proof of Theorem 4. We restrict $z$ and $s$ to $0<z<r_{1} \omega_{1}+\cdots+r_{k} \omega_{k}$ and $\operatorname{Re}(s)<1$ in Lemma 2.2. From Lemma 2.3 we can choose a sequence $T_{1} \leq \cdots \leq T_{N} \leq \cdots \rightarrow \infty$ such that

$$
\left|\frac{1}{\left(1-e^{-\omega_{1} t}\right)^{r_{1}} \cdots\left(1-e^{-\omega_{k} t}\right)^{r_{k}}}\right| \leq C
$$

for all $t=u \pm i T_{N}$ with $-1 \leq u \leq 1$, where $C$ is a constant depending only on $\omega_{1}, \ldots, \omega_{k}$ and $r_{1}, \ldots, r_{k}$. We put $T=T_{N}$ in Lemma 2.2. We estimate $\int_{C^{(7)}}$ and $\int_{C^{(8)}}$ on the left hand side of the equation in Lemma 2.2 with $T=T_{N}$ and take the limit as $N \rightarrow \infty$. First we treat $\int_{C(7)}$. We have

$$
\begin{aligned}
& \left|\int_{C^{(7)}}\right| \\
= & \left|\int_{\infty}^{-\infty} \frac{e^{-z\left(u+i T_{N}\right)}}{\left(1-e^{-\omega_{1}\left(u+i T_{N}\right)}\right)^{r_{1}} \cdots\left(1-e^{-\omega_{k}\left(u+i T_{N}\right)}\right)^{r_{k}}}\left(-u-i T_{N}\right)^{s-1} d u\right| \\
= & \left|\int_{|u|>1}+\int_{|u| \leq 1}\right| .
\end{aligned}
$$

It follows from $\operatorname{Re}(s)<1$ that $\left|\left(-u-i T_{N}\right)^{s-1}\right| \rightarrow 0$ as $N \rightarrow \infty$ uniformly in $u \in \mathbb{R}$. In addition, we have

$$
\begin{aligned}
& \left|\frac{e^{-z\left(u+i T_{N}\right)}}{\left(1-e^{-\omega_{1}\left(u+i T_{N}\right)}\right)^{r_{1}} \cdots\left(1-e^{-\omega_{k}\left(u+i T_{N}\right)}\right)^{r_{k}}}\right| \\
& \quad \leq \frac{e^{-z u}}{\left|1-e^{-\omega_{1} u}\right|^{r_{1}} \cdots\left|1-e^{-\omega_{k} u}\right|^{r_{k}}}
\end{aligned}
$$

and since $0<z<r_{1} \omega_{1}+\cdots+r_{k} \omega_{k}$ the right hand side above is integrable on $|u|>1$. These imply that $\left|\int_{|u|>1}\right| \rightarrow 0$ as $N \rightarrow \infty$. In the same manner together with (2.14), $\left|\int_{|u| \leq 1}\right| \rightarrow 0$ as $N \rightarrow \infty$. Hence (2.15) tends to 0 as $N \rightarrow \infty$. In the same manner we show that $\left|\int_{C^{(8)}}\right| \rightarrow 0$ as $N \rightarrow \infty$. Therefore

$$
\begin{aligned}
& \zeta_{r}(s, z, \underline{\omega})=-\Gamma(1-s) \lim _{N \rightarrow \infty} \sum_{j=1}^{k} \frac{(-1)^{r_{j}}}{\omega_{j}} \\
& \times \sum_{\substack{|n|<\omega_{j} T_{N} / 2 \pi \\
n \neq 0}}\left(\frac{2 \pi i n}{\omega_{j}}\right)^{s-1}\left[g_{r_{j}}\left(\frac{1}{2 \pi i n} \frac{\partial}{\partial u}\right)\left(\frac{u^{s-1} e\left(\frac{n z}{\omega_{j}} u\right)}{\prod_{l \neq j}\left(1-e\left(\frac{n \omega_{l}}{\omega_{j}} u\right)\right)^{r_{l}}}\right)\right]_{u=1} .
\end{aligned}
$$

Finally, we show that the summations over $n$ converge absolutely in $\operatorname{Re}(s)$ $<A$ for some $A \in \mathbb{R}$ as $N \rightarrow \infty$. From (2.12) it is sufficient to prove that 
for any $j$,

$$
\begin{array}{r}
\sum_{n \in \mathbb{Z} \backslash\{0\}}|n|^{\sigma-1}\left|\left[\frac{d^{m_{2}}}{d u^{m_{2}}}\left(\frac{1}{\prod_{l \neq j}\left(1-e\left(\frac{n \omega_{l}}{\omega_{j}} u\right)\right)^{r_{l}}}\right)\right]_{u=1}\right| \frac{1}{|n|^{m_{2}+m_{3}}} \\
\left(m_{2}, m_{3} \in \mathbb{Z}_{\geq 0}, m_{2}+m_{3} \leq r_{j}-1\right)
\end{array}
$$

converges for $\sigma<A$. By induction on $N$, for any $N, M \in \mathbb{Z}_{\geq 0}$ and $\alpha \in \mathbb{R}$ we easily obtain

$$
\frac{d^{N}}{d u^{N}}\left(\frac{1}{(1-e(\alpha u))^{M}}\right)=(2 \pi i \alpha)^{N} \sum_{l=0}^{N} \frac{b_{M, N, l}}{(1-e(\alpha u))^{M+l}}
$$

for some $b_{M, N, l} \in \mathbb{C}$. Hence, by the Leibniz rule, (2.16) is bounded above by the finite linear combination of

$$
\begin{aligned}
\sum_{n \in \mathbb{Z} \backslash\{0\}} \frac{|n|^{\sigma-1-m_{3}}}{\prod_{l \neq j}\left|1-e\left(n \omega_{l} / \omega_{j}\right)\right|^{r_{l}+N_{l}}} \\
\quad\left(N_{l}, m_{3} \in \mathbb{Z}_{\geq 0}, m_{3}+\sum_{l \neq j} N_{l} \leq r_{j}-1\right) .
\end{aligned}
$$

Since $\sin (\pi x) \geq 2 x$ for $0 \leq x \leq 1 / 2$, we have

$$
\begin{aligned}
\left|1-e\left(n \omega_{l} / \omega_{j}\right)\right| & =2\left|\sin \left(\pi|n| \omega_{l} / \omega_{j}\right)\right| \\
& =2 \sin \left(\pi|||n| \omega_{l} / \omega_{j} \|\right) \\
& \geq 4\left\||n| \omega_{l} / \omega_{j}\right\| .
\end{aligned}
$$

Applying this and the assumption $\left\||n| \omega_{l} / \omega_{j}\right\|^{-1}=O\left(|n|^{c_{j, l}}\right)$ to $(2.17)$, we finish the proof.

Proof of Theorem 3. First we prove (1). We assume $\operatorname{Im}(z)>0$ in addition to the assumptions of Lemma 2.2. Replacing $z$ with $r_{1} \omega_{1}+\cdots+r_{k} \omega_{k}-z$ in Lemma 2.2, we have

$$
\begin{aligned}
\frac{1}{2 \pi i}\left(-\int_{C_{\varepsilon}}+\right. & \left.\int_{C^{(7)}}+\int_{C^{(8)}}\right) \frac{e^{-\left(r_{1} \omega_{1}+\cdots+r_{k} \omega_{k}-z\right) t}}{\left(1-e^{-\omega_{1} t}\right)^{r_{1}} \cdots\left(1-e^{-\omega_{k} t}\right)^{r_{k}}}(-t)^{s-1} d t \\
= & -\sum_{j=1}^{k} \frac{(-1)^{r_{j}}}{\omega_{j}} \sum_{\substack{|n|<\omega_{j} T / 2 \pi \\
n \neq 0}}\left(\frac{2 \pi i n}{\omega_{j}}\right)^{s-1} \\
& \times\left[g_{r_{j}}\left(\frac{1}{2 \pi i n} \frac{\partial}{\partial u}\right)\left(\frac{u^{s-1} e\left(n r_{j} u\right) e\left(-\frac{n z}{\omega_{j}} u\right)}{\prod_{l \neq j}\left(e\left(-\frac{n \omega_{l}}{\omega_{j}} u\right)-1\right)^{r_{l}}}\right)\right]_{u=1}
\end{aligned}
$$


First we treat $\int_{C^{(7)}}$ on the left hand side of (2.18). We have

$$
\begin{aligned}
& \int_{C^{(7)}} \frac{e^{-\left(r_{1} \omega_{1}+\cdots+r_{k} \omega_{k}-z\right) t}}{\left(1-e^{-\omega_{1} t}\right)^{r_{1}} \cdots\left(1-e^{-\omega_{k} t}\right)^{r_{k}}}(-t)^{s-1} d t \\
& =\int_{C^{(7)}} \frac{e^{z t}}{\left(e^{\omega_{1} t}-1\right)^{r_{1}} \cdots\left(e^{\omega_{k} t}-1\right)^{r_{k}}}(-t)^{s-1} d t \quad(-\pi<\arg (-t)<0) \\
& =(-1)^{r-1} \int_{C^{(8)}} \frac{e^{-z t}}{\left(1-e^{-\omega_{1} t}\right)^{r_{1}} \cdots\left(1-e^{-\omega_{k} t}\right)^{r_{k}}} t^{s-1} d t \quad(-\pi<\arg (t)<0) \\
& =(-1)^{r} e^{-\pi i s} \int_{C^{(8)}} \frac{e^{-z t}}{\left(1-e^{-\omega_{1} t}\right)^{r_{1}} \cdots\left(1-e^{-\omega_{k} t}\right)^{r_{k}}}(-t)^{s-1} d t \\
& (0<\arg (-t)<\pi) .
\end{aligned}
$$

Similarly we have

$$
\begin{aligned}
& \int_{C^{(8)}} \frac{e^{-\left(r_{1} \omega_{1}+\cdots+r_{k} \omega_{k}-z\right) t}}{\left(1-e^{-\omega_{1} t}\right)^{r_{1}} \cdots\left(1-e^{-\omega_{k} t}\right)^{r_{k}}}(-t)^{s-1} d t \\
&=(-1)^{r} e^{\pi i s} \int_{C^{(7)}} \frac{e^{-z t}}{\left(1-e^{-\omega_{1} t}\right)^{r_{1}} \cdots\left(1-e^{-\omega_{k} t}\right)^{r_{k}}}(-t)^{s-1} d t \\
& \quad(-\pi<\arg (-t)<0) .
\end{aligned}
$$

Next we deal with the right hand side of (2.18). Replacing $n$ with $-n$, we have

$$
\begin{aligned}
& \sum_{\substack{|n|<\omega_{j} T / 2 \pi \\
n \neq 0}}\left(\frac{2 \pi i n}{\omega_{j}}\right)^{s-1}\left[g_{r_{j}}\left(\frac{1}{2 \pi i n} \frac{\partial}{\partial u}\right)\left(\frac{u^{s-1} e\left(n r_{j} u\right) e\left(-\frac{n z}{\omega_{j}} u\right)}{\prod_{l \neq j}\left(e\left(-\frac{n \omega_{l}}{\omega_{j}} u\right)-1\right)^{r_{l}}}\right)\right]_{u=1} \\
& =\sum_{\substack{|n|<\omega_{j} T / 2 \pi \\
n \neq 0}}\left(-\frac{2 \pi i n}{\omega_{j}}\right)^{s-1}\left[g_{r_{j}}\left(-\frac{1}{2 \pi i n} \frac{\partial}{\partial u}\right)\left(\frac{u^{s-1} e\left(-n r_{j} u\right) e\left(\frac{n z}{\omega_{j}} u\right)}{\prod_{l \neq j}\left(e\left(\frac{n \omega_{l}}{\omega_{j}} u\right)-1\right)^{r_{l}}}\right)\right]_{u=1} .
\end{aligned}
$$

It follows from $\arg \left(-2 \pi i n / \omega_{j}\right)= \pm \pi / 2$ that

$$
\left(-\frac{2 \pi i n}{\omega_{j}}\right)^{s-1}=-\left(\frac{2 \pi i n}{\omega_{j}}\right)^{s-1} \times \begin{cases}e^{-\pi i s} & \text { if } n>0, \\ e^{\pi i s} & \text { if } n<0,\end{cases}
$$

where $\arg \left(2 \pi i n / \omega_{j}\right)= \pm \pi / 2$.

Next we deal with $[\cdots]_{u=1}$ on the right hand side of (2.21). We need the following lemma: 
Lemma 2.4. Let $N \in \mathbb{Z}_{\geq 1}$ and $n \in \mathbb{Z} \backslash\{0\}$. Let a function $F(u)$ be a $(N-1)$-times continuously differentiable near $u=1$. Then

$$
\begin{aligned}
{\left[g_{N}\left(-\frac{1}{2 \pi i n} \frac{\partial}{\partial u}\right)(e(-n N u) F(u))\right]_{u=1} } & (-1)^{N-1}\left[g_{N}\left(\frac{1}{2 \pi i n} \frac{\partial}{\partial u}\right) F(u)\right]_{u=1}
\end{aligned}
$$

Proof. We define $a_{N, m}$ as in (2.10). Then the left hand side is calculated as follows:

$$
\begin{aligned}
{\left[g_{N}\left(-\frac{1}{2 \pi i n} \frac{\partial}{\partial u}\right)(e(-n N u) F(u))\right]_{u=1} } \\
\quad=\sum_{m=0}^{N-1} a_{N, m}\left(-\frac{1}{2 \pi i n}\right)^{m}\left[\frac{\partial^{m}}{\partial u^{m}}(e(-n N u) F(u))\right]_{u=1} \\
=\sum_{m=0}^{N-1} a_{N, m}\left(-\frac{1}{2 \pi i n}\right)^{m} \sum_{l=0}^{m}\left(\begin{array}{c}
m \\
l
\end{array}\right)(-2 \pi i n N)^{m-l} F^{(l)}(1) \\
=\sum_{l=0}^{N-1} \frac{F^{(l)}(1)}{l !}\left(-\frac{1}{2 \pi i n}\right)^{l}\left(\sum_{m=l}^{N-1} a_{N, m} \frac{m !}{(m-l) !} N^{m-l}\right) \\
=\sum_{l=0}^{N-1} \frac{F^{(l)}(1)}{l !}\left(-\frac{1}{2 \pi i n}\right)^{l} g_{N}^{(l)}(N) .
\end{aligned}
$$

Since $g_{N}(N-z)=(-1)^{N-1} g_{N}(z)$ we have

$$
(-1)^{l} \frac{g_{N}^{(l)}(N)}{l !}=(-1)^{N-1} \frac{g_{N}^{(l)}(0)}{l !}=(-1)^{N-1} a_{N, l}
$$

Applying this to (2.23) completes the proof.

Continuation of the proof of Theorem 3. From Lemma 2.4 we have

$$
\begin{gathered}
{\left[g_{r_{j}}\left(-\frac{1}{2 \pi i n} \frac{\partial}{\partial u}\right)\left(\frac{u^{s-1} e\left(-n r_{j} u\right) e\left(\frac{n z}{\omega_{j}} u\right)}{\prod_{l \neq j}\left(e\left(\frac{n \omega_{l}}{\omega_{j}} u\right)-1\right)^{r_{l}}}\right)\right]_{u=1}} \\
=(-1)^{r-1}\left[g_{r_{j}}\left(\frac{1}{2 \pi i n} \frac{\partial}{\partial u}\right)\left(\frac{u^{s-1} e\left(\frac{n z}{\omega_{j}} u\right)}{\prod_{l \neq j}\left(1-e\left(\frac{n \omega_{l}}{\omega_{j}} u\right)\right)^{r_{l}}}\right)\right]_{u=1}
\end{gathered}
$$


Applying (2.19)-(2.22) and (2.24) to (2.18), we obtain

$$
\begin{aligned}
& -\frac{1}{2 \pi i} \int_{C_{\varepsilon}} \frac{e^{-\left(r_{1} \omega_{1}+\cdots+r_{k} \omega_{k}-z\right) t}}{\left(1-e^{-\omega_{1} t}\right)^{r_{1}} \cdots\left(1-e^{-\omega_{k} t}\right)^{r_{k}}}(-t)^{s-1} d t \\
& \quad+\frac{1}{2 \pi i}\left((-1)^{r} e^{\pi i s} \int_{C^{(7)}}+(-1)^{r} e^{-\pi i s} \int_{C^{(8)}}\right) \\
& \quad \times \frac{e^{-z t}}{\left(1-e^{-\omega_{1} t}\right)^{r_{1}} \cdots\left(1-e^{-\omega_{k} t}\right)^{r_{k}}}(-t)^{s-1} d t \\
& =(-1)^{r-1} \sum_{j=1}^{k} \frac{(-1)^{r_{j}}}{\omega_{j}}\left(e^{-\pi i s} \sum_{0<n<\omega_{j} T / 2 \pi}+e^{\pi i s} \sum_{-\omega_{j} T / 2 \pi<n<0}\right) \\
& \quad \times\left(\frac{2 \pi i n}{\omega_{j}}\right)^{s-1}\left[g_{r_{j}}\left(\frac{1}{2 \pi i n} \frac{\partial}{\partial u}\right)\left(\frac{u^{s-1} e\left(\frac{n z}{\omega_{j}} u\right)}{\prod_{l \neq j}\left(1-e\left(\frac{n \omega_{l}}{\omega_{j}} u\right)\right)^{r_{l}}}\right)\right]_{u=1} .
\end{aligned}
$$

Multiplying the equation of Lemma 2.2 by $e^{\pi i s} \Gamma(1-s)$ and $(-1)^{r-1} \Gamma(1-s)$ by (2.25) respectively and summing up these, we obtain

$$
\begin{aligned}
& e^{\pi i s} \zeta_{r}(s, z, \underline{\omega})+(-1)^{r-1} \zeta_{r}\left(s, r_{1} \omega_{1}+\cdots+r_{k} \omega_{k}-z, \underline{\omega}\right) \\
& +\frac{1}{\Gamma(s)} \int_{C^{(8)}} \frac{e^{-z t}}{\left(1-e^{-\omega_{1} t}\right)^{r_{1}} \cdots\left(1-e^{-\omega_{k} t}\right)^{r_{k}}}(-t)^{s-1} d t \\
& =-\frac{2 \pi i}{\Gamma(s)} \sum_{j=1}^{k} \frac{(-1)^{r_{j}}}{\omega_{j}} \\
& \quad \times \sum_{0<n<\omega_{j} T / 2 \pi}\left(\frac{2 \pi i n}{\omega_{j}}\right)^{s-1}\left[g_{r_{j}}\left(\frac{1}{2 \pi i n} \frac{\partial}{\partial u}\right)\left(\frac{u^{s-1} e\left(\frac{n z}{\omega_{j}} u\right)}{\prod_{l \neq j}\left(1-e\left(\frac{n \omega_{l}}{\omega_{j}} u\right)\right)^{r_{l}}}\right)\right]_{u=1} .
\end{aligned}
$$

Here, we used the formula

$$
\left(e^{\pi i s}-e^{-\pi i s}\right) \Gamma(1-s)=2 i \sin (\pi s) \Gamma(1-s)=2 i \frac{\pi}{\Gamma(s) \Gamma(1-s)} \Gamma(1-s)=\frac{2 \pi i}{\Gamma(s)} .
$$

By the method similar to the first part of the proof of Theorem 4, there exists a sequence $T_{1} \leq \cdots \leq T_{N} \leq \cdots \rightarrow \infty$ such that

$$
\begin{aligned}
& e^{\pi i s} \zeta_{r}(s, z, \underline{\omega})+(-1)^{r-1} \zeta_{r}\left(s, r_{1} \omega_{1}+\cdots+r_{k} \omega_{k}-z, \underline{\omega}\right) \\
& =-\frac{2 \pi i}{\Gamma(s)} \lim _{N \rightarrow \infty} \sum_{j=1}^{k}(-1)^{r_{j}} / \omega_{j} \\
& \quad \times \sum_{0<n<\omega_{j} T_{N} / 2 \pi}\left(\frac{2 \pi i n}{\omega_{j}}\right)^{s-1}\left[g_{r_{j}}\left(\frac{1}{2 \pi i n} \frac{\partial}{\partial u}\right)\left(\frac{u^{s-1} e\left(\frac{n z}{\omega_{j}} u\right)}{\prod_{l \neq j}\left(1-e\left(\frac{n \omega_{l}}{\omega_{j}} u\right)\right)^{r_{l}}}\right)\right]_{u=1} .
\end{aligned}
$$


By the method similar to the last part of the proof of Theorem 4 together with $\left|e\left(n z / \omega_{j}\right)\right|=e^{-2 \pi n \operatorname{Im}(z) / \omega_{j}}$, the summations over $n$ converge absolutely and uniformly on any compact subset of $\left\{(s, z) \in \mathbb{C}^{2}: \operatorname{Im}(z)>0\right\}$ when $N \rightarrow \infty$. This completes the proof of (1).

(2) is easily obtained by replacing $z$ with $r_{1} \omega_{1}+\cdots+r_{k} \omega_{k}-z$ in (1) and applying Lemma 2.4 .

\section{The generalized multiple sine functions and absolute tensor} products. In this section we prove Theorems 1 and 2 .

Proof of Theorem 2. (1) We differentiate the equation of Theorem 3(1) with respect to $s$ at $s=-m$. Since

$$
\begin{aligned}
{\left[\frac{1}{\Gamma(s)}\right]_{s=-m} } & =0 \\
{\left[\frac{d}{d s}\left(\frac{1}{\Gamma(s)}\right)\right]_{s=-m} } & =\lim _{s \rightarrow-m} \frac{1}{(s+m) \Gamma(s)}=\lim _{s \rightarrow-m} \frac{s(s+1) \cdots(s+m-1)}{\Gamma(s+m+1)} \\
& =(-1)^{m} m !
\end{aligned}
$$

we obtain (1).

(2) Multiplying the equation of Theorem 3(2) by $e^{-\pi i s}$ and differentiating with respect to $s$ at $s=-m$, we obtain (2).

Proof of Theorem 1. From [A2, Lemma 4.1] we have

$$
\zeta\left(s, \mathbb{F}_{p_{1}}\right)^{\otimes r_{1}} \otimes \cdots \otimes \zeta\left(s, \mathbb{F}_{p_{k}}\right)^{\otimes r_{k}} \cong S_{r}(i s, \underline{\omega})^{(-1)^{r}}
$$

where

$$
\underline{\omega}:=(\underbrace{\frac{2 \pi}{\log p_{1}}, \ldots, \frac{2 \pi}{\log p_{1}}}_{r_{1} \text { copies }}, \ldots, \underbrace{\frac{2 \pi}{\log p_{k}}, \ldots, \frac{2 \pi}{\log p_{k}}}_{r_{k} \text { copies }}) .
$$

Then $\underline{\omega}$ satisfies the condition of Theorem 2 (see Remark 1.1). Applying Theorem 2(1) with $m=0$ to (3.1), we finish the proof.

Added in proof (June, 2006). Our method and result are extendable to other cases; cf. also A. Narukawa, The modular properties and the integral representations of the multiple elliptic gamma functions, Adv. Math. 189 (2004), 247-267. We have generalized our result to absolute tensor products of the Riemann zeta function in: H. Akatsuka, The double Riemann zeta function, preprint, June 2006.

\section{References}

[A1] H. Akatsuka, Euler product expression of triple zeta functions, Internat. J. Math. 16 (2005), 111-136.

[A2] -, Degeneration of multiple Euler products, Acta Arith. 121 (2006), 63-91.

[B] A. Baker, Transcendental Number Theory, Cambridge Univ. Press, 1975. 
[Bar] E. W. Barnes, On the theory of the multiple gamma functions, Trans. Cambridge Philos. Soc. 19 (1904), 374-425.

[K] N. Kurokawa, Multiple zeta functions: an example, in: Zeta Functions in Geometry (Tokyo, 1990), Adv. Stud. Pure Math. 21, Kinokuniya, Tokyo, 1992, 219-226.

[KK1] N. Kurokawa and S. Koyama, Multiple sine functions, Forum Math. 15 (2003), 839-876.

[KK2] -, 一, Kummer's formula for multiple gamma functions, J. Ramanujan Math. Soc. 18 (2003), 87-107.

[KK3] - - - Multiple zeta functions: the double sine function and the signed double Poisson summation formula, Compos. Math. 140 (2004), 1176-1190.

[KK4] -, -, Multiple Euler products, Tr. St.-Peterburg. Mat. Obshch. 11 (2005), 123166 (in Russian); English transl. will be published in Amer. Math. Soci. Transl.

[KO] N. Kurokawa and H. Ochiai, Generalized Kinkelin's formulas, preprint, 2005.

[KW1] N. Kurokawa and M. Wakayama, Absolute tensor products, Int. Math. Res. Not. 2004, no. 5, 249-260.

[KW2] -, -, Period deformations and Raabe's formulas for generalized gamma and sine functions, preprint, 2005.

[M] Y. Manin, Lectures on zeta functions and motives (according to Deninger and Kurokawa), Astérisque 228 (1995), 121-163.

[N] N. Nörlund, Vorlesungen über Differenzenrechnung, Chelsea, 1954.

Department of Mathematics

Tokyo Institute of Technology

2-12-1 Ookayama, Meguro-ku

Tokyo, 152-8551, Japan

E-mail: akatsuka@math.titech.ac.jp

Received on 12.1.2006

and in revised form on 15.3.2006 